\title{
La retroalimentación y su influencia en la consolidación de aprendizajes en proceso, de niños y niñas de Educación
}

\section{Inicial.}

Feedback and its influence on the consolidation of learning in process, of boys and girls of Initial Education

Allison Michelle Llerena Espín. ${ }^{1}$, Irelys Sánchez Fernández. ${ }^{2}$ \& Yoel Hernández Navarro. ${ }^{3}$

Recibido:05-06-2020 / Revisado: 25-07-2020 /Aceptado: 18-08-2020/ Publicado: 04-09-2020

\begin{abstract}
.
DOI: https://doi.org/10.33262/concienciadigital.v3i3.2.1408

The main objective is to investigate how the feedback process together with the parents, helps to consolidate learning processes, thus being able to prevent children from feeling frustrated about not obtaining an apprenticeship that the rest has already achieved. This project used a quantitative approach to have exact meanings of what is being presented, with a population of 65 children of Maternal 1 and 2 of the University Child Development Center to whom an observation sheet and 6 teachers of Maternal 1 were applied and 2 with those who worked with a survey questionnaire to collect accurate data that served to perform the corresponding analysis. Similarly, the hypothesis verification was carried out by means of Kendall's Tau_b statistic, which resulted in its validation. It explains precisely how the objectives set out in the research project were met. Finally, conclusions and research recommendations will be provided.
\end{abstract}

\footnotetext{
${ }^{1}$ Centro médico "Corfint”.,Ambato, Ecuador, allimichelle1997@ hotmail.com

${ }^{2}$ Universidad Técnica de Ambato, Facultad de Ciencias Humanas y de la Educación (FCHE), Ambato, Ecuador, i.sanchez@uta.edu.ec., iD https://orcid.org/0000-0003-4075-6008

${ }^{3}$ Universidad Técnica de Ambato, Facultad de Ciencia e Ingeniería en Alimentos y Biotecnología (FCIAB), Ambato, Ecuador, yoel.hernandez@uta.edu.ec(iD) https://orcid.org/0000-0002-0178-4949
} 
Keywords: Consolidation, feedback, communication, integral development.

\section{Resumen.}

El objetivo central es investigar cómo el proceso de retroalimentación en conjunto con los padres ayuda a la consolidación de aprendizajes en procesos, pudiendo de esta manera evitar que los niños y niñas se sientan frustrados en cuanto a no obtener un aprendizaje que el resto ya consiguió. Este proyecto utilizó un enfoque cuantitativo para tener acepciones exactas de lo que se está presentando, con una población de 65 niños y niñas de Educación Inicial, a quienes se aplicó una ficha de observación y sus 6 docentes, a quienes se les aplicó un cuestionario para recolectar datos precisos que sirvieron para efectuar el análisis correspondiente. De igual manera, se realizó la verificación de hipótesis por medio del estadístico Tau_b de Kendall, dando como resultado su validación. Se explica con precisión cómo se cumplieron los objetivos planteados en el proyecto de investigación. Para finalizar se brindan conclusiones y recomendaciones de la investigación.

Palabras claves: Retroalimentación, consolidación, comunicación, desarrollo integral.

\section{Introducción}

La retroalimentación juega un papel importante dentro del proceso de enseñanza aprendizaje de los niños y niñas, vinculado con la necesidad de que los maestros logren un aprendizaje significativo, que sea de gran aporte para sus estudiantes con la ayuda de los padres de familia que lo reforzarán en el hogar. Además, se debe tener en cuenta como metodología básica el análisis y síntesis de contenidos, los cuales permiten alcanzar razonamientos válidos (Cedeño, 2019).

Para Gallego, Ospina, Quintero, \& Jaramillo (2015), los docentes contribuyen a la formación integral de los niños de preescolar, considerando sus necesidades, etapas de desarrollo y sus diferentes dimensiones. De esta manera, deberían lograr que los aprendizajes se consoliden dentro del aula de clases, estableciendo las posibles pautas de lo que se debe realizar para evitar vacíos de comprensión en los niños y niñas y aportando sus conocimientos al proceso de enseñanza aprendizaje.

En este sentido es necesario tomar en consideración lo que manifiesta Contreras \& Zuñiga (2017), al plantear que dentro de la retroalimentación se encuentra la evaluación formativa, la cual se basa 
en el análisis de lo que sucede dentro del aula y de las diferentes recepciones de aprendizajes de los niños y niñas en el momento de adquirir los conocimientos. Se realiza una indagación sobre las falencias, para de esta manera, concluir, tomar decisiones, realizar una eficaz enseñanza y consolidar aprendizajes que se encuentran con vacíos de comprensión.

Asimismo, la retroalimentación ayuda a los niños y niñas a cambiar o corregir su pensamiento y mejorar su aprendizaje, permitiéndoles a los docentes adaptar la enseñanza con el objetivo de lograr una mejora continua a lo largo del proceso de aprendizaje (Margalef, 2017).

Por otra parte, la consolidación de aprendizajes es un elemento también importante a tener en cuenta en el proceso de enseñanza aprendizaje. Es todo aquello que la docente realiza para que el conocimiento llegue a los niños, como fortalecimiento de un aprendizaje, es decir, abarca las estrategias, métodos y formas de enseñanza, con la ayuda de los padres de familia y asegurando en casa el aprendizaje mediante un recuento de lo realizado en el aula de clases, de esta forma, el entendimiento no quedaría retrasado y todo marcharía a la par con la enseñanza docente (Trujillo, 2013).

En este mismo sentido, Osorio \& Alexis (2014), plantean que la retroalimentación y la consolidación de aprendizajes están estrechamente relacionados, pues con ambos se logra un proceso eficaz de enseñanza aprendizaje. Al momento de realizar la retroalimentación se consolidan aquellos conocimientos que no quedaron claros y se alcanza, por parte de la docente y los padres de familia, un aprendizaje significativo.

Nos dice Razeto (2016) que lograr que los padres de familia se involucren en el proceso de enseñanza aprendizaje, ayuda a fortalecer el aprendizaje además de entender el desarrollo de los conocimientos y las habilidades de los niños durante el periodo escolar. Mediante la comunicación que establecerán con la docente se verán prestos a ayudar al refuerzo que darán en el hogar, para así favorecer el desarrollo integral de sus hijos.

Por lo tanto, Frontela (2013) manifiesta que el éxito de la docente es mantener una comunicación favorable con la familia de los niños y niñas, pues al crear un ambiente adecuado, el aprendizaje 
se verá solidificado de mejor manera, ya que en casa se reforzarán los conocimientos que serán impartidos en el aula de clases, pues es un trabajo conjunto que permitirá llegar a un incremento de relaciones efectivas. Para ello, el docente debe tener una serie de características que ayuden al desenvolvimiento de los niños y niñas de una manera espontánea, que les permita adquirir los conocimientos esenciales para su mejor desarrollo. (Llerena, 2020)

De esta manera, se debe crear un ambiente favorable para los niños y niñas, que influya de manera positiva en su desarrollo, crecimiento y exploración. Se debe tener en cuenta que el involucramiento de los padres de familia es esencial, pues serán ellos quienes favorezcan el desarrollo pleno, el trabajo mutuo para potenciar las habilidades y formar personas aptas para la vida, brindándoles relaciones seguras que les ayude a desenvolverse de manera eficaz. (Sánchez, 2004)

El rol docente debe basarse en lograr una instrucción centrada en los niños y niñas, desarrollándole determinadas capacidades como: ser críticos, seres pensantes, obtener de ellos su mayor potencial, apoyándolos con los conocimientos que requieren para su vida diaria, además de brindarles diversas percepciones que los ayuden a tener una variada perspectiva, para construir su propio conocimiento y promover su desarrollo personal (León, 2013).

Es importante destacar, además, que, para consolidar aprendizajes influye mucho el método de enseñanza que la maestra utiliza, como, por ejemplo, indagar conocimientos previos para fortalecerlos o comenzar a enseñarlos. De esta manera, se imparte un nuevo conocimiento y los métodos que utilice la maestra serán de fundamental importancia para construcción de este, por eso es de vital importancia el papel de la maestra al momento de crear nuevos aprendizajes y de fortalecerlos cada día, hasta que queden afianzados por completo. (Mejía, 2014, citado en Llerena, 2020)

Los padres de familia deben estar involucrados dentro del trabajo colaborativo que realiza la docente con sus estudiantes, además que la docente debe tener una buena comunicación para poder indicar actividades que refuercen el conocimiento, según Martínez, Guevara, \& Valles (2016, 
citado en Llerena, 2020). Se necesita una participación efectiva y activa de los padres de familia, para que se involucren y trabajen colaborativamente para lograr un mismo objetivo, el cual se verá reflejado dentro del informe cualitativo descriptivo. (Martínez, Guevara, \& Valles, 2016, citado en Llerena, 2020)

Para garantizar el éxito dentro del aprendizaje se debe procurar la participación activa de los padres de familia, la docente es la encargada de lograr que ellos se vean involucrados, para alcanzar una meta en común, que sería la consolidación de los aprendizajes en niños y niña. (Blanco, 2019, citado en Llerena, 2020)

En esta dirección, Cárdenas \& Pastrana (2016, citado en Llerena, 2020) nos hablan sobre las experiencias educativas que necesitan de consolidaciones de aprendizaje, pues pueden ser desfavorable en posteriores conocimientos. El aprendizaje consta de una serie de indicadores que permitan resolver aquella falta de comprensión de los niños y niñas, donde el maestro debe buscar la estrategia para que se pueda llegar a una comprensión plena, que es una de las características que la docente debe tener para impartir sus conocimientos.

Según Llerena (2020), la consolidación de aprendizajes da un realce cuando se toman en cuenta los conocimientos previos de los niños y niñas y lo empiezan a utilizar como el punto de partida para solidificar el mismo. Además, las estrategias empleadas harán que los aprendizajes mejoren mediante la observación, asumiendo la responsabilidad del propio aprendizaje por medio de la ayuda docente y de los padres de familia, quienes fortalecerán y orientarán para que se logre el objetivo a cumplir. Para que no exista un desequilibrio en el aprendizaje de los niños y niñas, la docente debe tener en cuenta las habilidades de los niños y desarrollar actividades que permitan la consolidación de aprendizajes de manera adecuada, sin que queden vacíos de compresión, partiendo desde lo más sencillo hacia lo más complejo (Gómez, 2014).

Con base al análisis científico, se determina como objetivo investigar cómo influye el proceso de retroalimentación realizado por los docentes en conjunto con los padres de familia, en la consolidación de aprendizajes en procesos en niños y niñas de Educación Inicial. 


\section{Metodología}

Este estudio con un enfoque cuantitativo y un diseño correlacional tuvo el propósito de evaluar el grado de incidencia entre las variables utilizadas en el problema de investigación: la independiente, que se refiere a la retroalimentación y la dependiente, que es la consolidación de aprendizajes en procesos. (Llerena, 2020). Se utilizó el SPSS (Statistical Package for the Social Sciences), un programa que permitió realizar análisis estadísticos y la validación de los instrumentos utilizados. Para la validación de hipótesis se utilizó Tau_b de Kendall, el cual ayudó al proceso estadístico de la investigación. Para la recolección de información, las técnicas utilizadas fueron la observación y la encuesta, con sus respectivos instrumentos; ficha de observación estructurada con 5 ítems, dirigida a 65 niños y niñas de Educación Inicial; y un cuestionario conformado por 5 preguntas cerradas, aplicado a 6 docentes. En total, se trabajó con 71 personas del CDI Centro de Desarrollo Infantil Universitario UTA.

\section{Resultados de la ficha de observación:}

El 100\% de las docentes indaga conocimientos previos antes de empezar a impartir su clase, pues al momento de conocer a los niños y niñas no se puede saber los conocimientos adquiridos en el hogar. (Llerena, 2020). Para Silva (2014, citado en Llerena, 2020), las docentes indagan conocimientos previos para poder nivelar de esta manera a los niños y niñas en cuanto a saberes, lo que está vinculado con lo observado antes de iniciar un tema de clases, donde las docentes investigan para saber cuánto saben del tema a tratar.

El $98 \%$ de las docentes realiza preguntas que ayudan a solventar dudas pues mediante las mismas se da la consolidación de aprendizajes. Se puede deducir que la mayor parte se interesa en que los niños y niñas adquieran el conocimiento y que sea duradero mediante la realización de estas preguntas, pues de esta manera los niños y niñas no se quedan con vacíos de comprensión y la maestra debe estar siempre dispuesta a responder con efectividad para que el aprendizaje que recibieron se consolide. (Llerena, 2020). Lima (2017) considera que la consolidación de aprendizajes se logra mediante preguntas que ayuden a la retroalimentación de un tema tratado, 
pues crea un impacto importante en el aprendizaje y ayuda a tomar control de su propio aprendizaje haciéndolo más efectivo.

Del total de 65 niños y niñas que pertenece al 100\% de la muestra, el $62 \%$ responde a preguntas realizadas por la docente en cuanto al tema tratado en clase, mientras que el $29 \%$ a veces contestan, en ocasiones por falta de atención o porque no entendieron y un $9 \%$ nunca responde debido a la escasez de vocabulario o falta de estimulación recibida por parte de las docentes y los padres de familia. (Llerena, 2020)

Polanco (2004), hace referencia a que las preguntas ayudan a formar niños y niñas críticos, reflexivos y creativos, ya que fomentan en los niños su capacidad de reflexión acerca del tema tratado en clases, por lo que es de suma importancia realizar preguntas pedagógicas para fomentar el conocimiento.

Del 100\% que corresponde al 65 de niños y niñas, el $42 \%$ siempre describe el tema tratado en clase sin dificultad, mientras que el $35 \%$ a veces lo describe pues tienen dificultades en el vocabulario y el 23\% nunca responde al tema tratado, ya que no existe una apropiada estimulación al vocabulario, teniendo en cuenta que es una edad en la que están adquiriendo mucho vocabulario. (Llerena, 2020). Como plantea Cáceres (2018), la estimulación del lenguaje permite aprendizajes mejores y más significativos, además de servir como impulso constante hacia la lectura inicial y de comprender el significado de distintas palabras dando otra perspectiva al aprendizaje en los niños y niñas.

Un $99 \%$ de estos niños y niñas son reforzados por la maestra mediante el juego, pues esta actividad permite a los niños adquirir el aprendizaje requerido. Estos resultados permiten evidenciar que la actividad principal de los niños y niñas es el juego, por lo tanto, las docentes aplican esta estrategia para reforzar conocimientos sobre el tema tratado en clase, de esta manera se retroalimentan de igual forma los aprendizajes. Ospina (2015) manifiesta que el juego es una actividad que permite aumentar la creatividad, además promueve la curiosidad que les ayudará a descubrir el mundo que los rodea, incentivando el desarrollo adecuado del proceso de aprendizaje. 


\section{Resultados de la encuesta aplicada a las docentes:}

El 50\% de los docentes investigados trabaja conjuntamente con los padres de familia para realizar la retroalimentación, pues de esta manera se consolidan los aprendizajes que están en procesos, el $33 \%$ a veces trabaja con los padres de familia, aduciendo que es difícil la accesibilidad a estos, ya que en ocasiones no cuentan con el tiempo necesario y un $17 \%$ nunca trabaja con los padres de familia, ya que a los padres de familia solo les interesa el cuidado de sus hijos, pero no el aprendizaje en sí. Según Domínguez (2010), para el desarrollo de los niños y niñas debe existir una buena relación entre la docente y los padres de familia, pues ambos tienen un objetivo en común que es el desarrollo armónico e integral de los niños y para lograrlo se necesita de procesos de colaboración por parte de los padres de familia.

El 83\% de las docentes encuestadas siempre retroalimenta el trabajo individual de los niños y niñas ya que es fundamental que todos participen y refuercen lo que están fallando, el 17\% a veces realiza la retroalimentación individual ya que trabajan de manera colectiva mejor, pues les toma menos tiempo que de manera individual. Según Caballero (2017), la retroalimentación permite expresar opiniones sobre el proceso de aprendizaje, visualizar las fortalezas y debilidades de los niños y niñas, desarrollar ciertas capacidades que ayuden a formar niños y niñas competentes, para enfrentar acciones de la vida cotidiana.

El 50\% de las docentes respondieron que siempre plantean preguntas a los padres de familia que permitan verificar si el aprendizaje ha sido consolidado, mientras que el 33\% respondieron que a veces lo hacen puesto que los padres de familia no cuentan con el tiempo suficiente ni el compromiso para con los niños y niñas y un 17\% nunca lo hace por la misma razón que los padres de familia a veces no disponen de accesibilidad ni existe un espacio para lograr platicar sobre el tema. Para (Flórez, 2017) los niños y niñas necesitan claramente la atención de los padres de familia para poder lograr un aprendizaje equitativo, pues la familia es el eje fundamental para un desarrollo pleno en todas las instancias, los padres de familia deben tener en cuenta que para que un aprendizaje sea consolidado se necesita de la ayuda en casa porque es un trabajo mutuo. 
El 50\% de las docentes siempre plantean preguntas a los padres de familia, que permitan verificar si el aprendizaje ha sido consolidado, mientras que el $33 \%$ a veces lo hacen, pues los padres de familia no cuentan con el tiempo suficiente ni con el compromiso para con sus hijos y un 17\% nunca lo hace, porque los padres de familia a veces no disponen de accesibilidad, ni existe un espacio para lograr platicar sobre el tema. Para Flórez (2017), los niños y niñas necesitan la atención de sus padres para lograr un aprendizaje equitativo, pues la familia es el eje fundamental para un desarrollo pleno en todas las instancias; los padres de familia deben tener en cuenta que para que un aprendizaje sea consolidado, se necesita de la ayuda en casa pues es un trabajo mutuo.

El $67 \%$ de estos docentes crea espacios de reflexión con los padres de familia que evidencien la consolidación de aprendizajes, mientras que el 33\% a veces lo hace, pues cuando los padres de familia son citados a reuniones, no asisten y no se pueden desarrollar espacios de reflexión sobre sus hijos. Para Domínguez (2010), la clave para generar una atención de los padres de familia es que vean la importancia de su participación para la educación de sus hijos, de esta manera los padres deben entender su influencia en la transmisión de valores y en todo lo que les rodea a los niños y niñas.

El 83\% de los docentes siempre dan instrucciones a los padres de familia para que retroalimenten el tema tratado en clase, el $17 \%$ a veces da instrucciones porque no siempre son los padres de familia los que van a retirar a los niños de la institución, o no cuentan con el tiempo necesario pues la mayor parte trabaja y no es el espacio suficiente para poder prestar la atención necesaria. Según Rodríguez (2006), los niños que se sienten apoyados por los padres de familia y que reciben estimulación para su desarrollo integral, obtienen mejores resultados en todo lo que se propongan, en este sentido es la docente la encargada de transmitir a los padres de familia el objetivo de la educación y de sensibilizarlos.

\section{Análisis e interpretación final de los resultados:}

Silva (2014) y Lima (2017) ven claro que indagar conocimientos previos ayuda a fortalecer el tema tratado en clases, en el momento en el que la docente realiza preguntas para consolidar 
aprendizajes, serán respondidas por los niños de la mejor manera, asimismo mediante el juego, como plantea Ospina (2015), se refuerzan conocimientos que aún no son asimilados, aumentando así la creatividad y despertando la curiosidad en los niños y niñas.

De acuerdo con Flórez (2017), los resultados obtenidos nos permiten observar que las docentes no tienen la apertura suficiente para con los padres de familia y conversar sobre las falencias que tienen sus hijos, para de esta manera, retroalimentarlos en casa y poder consolidar de manera efectiva los aprendizajes. Para Rodríguez (2006), la mayor parte de los docentes les da a los padres de familia, las instrucciones para que retroalimenten el tema tratado en clase, pero no siempre estos se involucran, por lo cual no se logra la óptima consolidación de aprendizajes.

Según un análisis realizado por Silva (2014) y Caballero (2017), la retroalimentación y la consolidación de aprendizajes están netamente ligadas al aprendizaje de los niños y niñas, pues al realizar una retroalimentación con los padres de familia, se puede llegar al aprendizaje significativo, fortaleciéndose mediante la ayuda de estos.

No obstante, cabe destacar que la retroalimentación no siempre se puede lograr, por la falta de tiempo para los padres de familia y son ellos el eje fundamental del aprendizaje de sus hijos. Las maestras en el salón de clases son las responsables de consolidar los aprendizajes, sobre todo mediante el juego, ya que dentro de la institución están a cargo de confortar el aprendizaje que no se encuentra claro y de esta manera, los niños y niñas adquieran las destrezas correspondientes.

\section{Verificación de hipótesis:}

En este estudio se llevó a cabo el análisis de hipótesis, mediante estadísticos no paramétricos, que se ajustan a la verificación de la hipótesis. Se tomó en cuenta un grupo de 71 personas. (Llerena, 2020). De acuerdo con la prueba no paramétrica de Tau_b de Kendall de un grupo relacionada se obtuvieron los siguientes datos como lo muestra la tabla siguiente: 
Tabla Nº : Prueba de Tau_b de Kendall para un grupo de personas.

\begin{tabular}{|c|c|c|c|c|}
\hline & & & $\begin{array}{l}\text { ¿Los niños } \\
\text { responden a } \\
\text { preguntas realizadas } \\
\text { por la docente sobre } \\
\text { el tema principal? }\end{array}$ & $\begin{array}{l}\text { ¿Los niños describen sin } \\
\text { dificultad el tema tratado } \\
\text { en clases? }\end{array}$ \\
\hline \multirow[t]{15}{*}{ Tau_b de kendall } & \multirow{3}{*}{$\begin{array}{l}\text { ¿Trabaja } \\
\text { conjuntamente con los } \\
\text { padres de familia para } \\
\text { realizar } \\
\text { retroalimentación? }\end{array}$} & $\begin{array}{l}\text { Coeficiente de } \\
\text { correlación }\end{array}$ &, 384 & , $498^{*}$ \\
\hline & & Sig. (bilateral) & ,085 & ,027 \\
\hline & & $\mathrm{N}$ & 20 & 20 \\
\hline & \multirow{3}{*}{$\begin{array}{ll}\text { ¿Retroalimenta } & \text { el } \\
\text { trabajo individual de } \\
\text { los niños y niñas? }\end{array}$} & $\begin{array}{l}\text { Coeficiente de } \\
\text { correlación }\end{array}$ &,- 110 &,- 167 \\
\hline & & Sig. (bilateral) & ,629 & ,468 \\
\hline & & $\mathrm{N}$ & 20 & 20 \\
\hline & \multirow{3}{*}{$\begin{array}{l}\text { ¿Plantea preguntas a } \\
\text { los padres de familia } \\
\text { que permitan verificar } \\
\text { si el aprendizaje ha } \\
\text { sido consolidado? }\end{array}$} & $\begin{array}{l}\text { Coeficiente de } \\
\text { correlación }\end{array}$ & $1,000^{* *}$ &, $658^{* *}$ \\
\hline & & Sig. (bilateral) &, 0 &, 004 \\
\hline & & $\mathrm{N}$ & 20 & 20 \\
\hline & \multirow{3}{*}{$\begin{array}{lr}\text { ¿Crea espacios } & \text { de } \\
\text { reflexión con los } \\
\text { padres de familia } & \text { que } \\
\text { evidencien } & \text { la } \\
\text { consolidación } & \text { de } \\
\text { aprendizajes? } & \end{array}$} & $\begin{array}{l}\text { Coeficiente de } \\
\text { correlación }\end{array}$ &, $658^{* *}$ & $1,000^{* *}$ \\
\hline & & Sig. (bilateral) &, 004 &, 0 \\
\hline & & $\mathrm{N}$ & 20 & 20 \\
\hline & \multirow{3}{*}{$\begin{array}{l}\text { ¿Da instrucciones a los } \\
\text { padres de familia para } \\
\text { que retroalimenten a } \\
\text { sus hijos el tema } \\
\text { tratado en clase? }\end{array}$} & $\begin{array}{l}\text { Coeficiente de } \\
\text { correlación }\end{array}$ &, 411 &, $667^{* *}$ \\
\hline & & Sig. (bilateral) &, 070 & ,004 \\
\hline & & $\mathrm{N}$ & 20 & 20 \\
\hline
\end{tabular}

Elaborado por. Grupo de investigación

Decisión: Los resultados del análisis de correlación permiten aceptar la hipótesis: "La mayor aplicación de retroalimentación consolida los aprendizajes en procesos en niños y niñas del Centro de Desarrollo Infantil Universitario UTA". Cuando se retroalimenta a padres de familia, la capacidad de describir el tema tratado en clases es mejor, pues son los que refuerzan el aprendizaje 
para que sea consolidado. Además, cuando la docente plantea preguntas que permiten verificar si el aprendizaje ha sido consolidado, la capacidad de responder aumenta y el tema tratado en clase es descrito con mayor facilidad. Asimismo, cuando la docente crea espacios de reflexión con los padres de familia que evidencien la consolidación de estos aprendizajes, estos toman conciencia acerca de lo que sus hijos están trabajando en el salón de clases y la capacidad de responder a preguntas planteadas por la docente será más efectiva.

Por último, al momento de dar instrucciones a los padres de familia para que retroalimenten a sus hijos en casa, ayudaría a que se fortalezcan los contenidos impartidos, conllevando a mejores resultados en los aprendizajes. (Llerena, 2020)

\section{Conclusiones}

- Es de destacar que la mitad de los docentes encuestados afirmó que siempre realiza la retroalimentación con los padres de familia, sin embargo, se caracteriza por ser general y escasa, pues los padres no disponen del tiempo necesario para dedicarle a este proceso. Los padres priorizan el cuidado de sus hijos y no les interesa las destrezas que ellos puedan alcanzar. Por tanto, se aprecia que no existe una relación cara a cara y efectiva de comunicación, de los docentes con los padres de familia, donde los padres dispongan del tiempo suficiente para explicarles las demandas y los aprendizajes adquiridos por sus hijos.

- La totalidad de la muestra coincide en que, para alcanzar la consolidación de los aprendizajes en procesos, se debe realizar una indagación de conocimientos antes de comenzar la clase planificada, resolver las dudas de los niños y niñas y reforzar su conocimiento, utilizando el juego, que es la actividad principal a través de la cual adquieren la mayor parte del conocimiento, convirtiéndose en el determinante principal para la consolidación de sus aprendizajes.

- Mediante Tau_b Kendall se pudo afirmar la correlación entre las dos variables analizadas: retroalimentación y consolidación de aprendizajes en procesos dando. La docente es la encargada de reforzar conocimientos que no se encuentren claros, trabajando de conjunto con los padres de familia, quienes son los primeros responsables de la educación de los niños y niñas, lográndose de esta manera, la consolidación de los aprendizajes. 


\section{Referencias Bibliográficas}

Blanco, V. (2014). Una propuesta de trabajo para implicar a las familias en las escuelas. Recuperado de https://rodin.uca.es/xmlui/bitstream/handle/10498/16607/16607.pdf

Caballero, A. (2017). La retroalimentación como estrategia didáctica para fortalecer las prácticas evaluativas en el aula escolar. Recuperado de: https://repository.usta.edu.co/bitstream/handle/11634/9513/CaballeroAdelina2017.pdf?seque nce=1\&isAllowed=y

Cáceres, M. (2018). Vocabulario receptivo en estudiantes de preescolar en la comunidad de Talca, Chile. Recuperado de: http://www.scielo.org.mx/pdf/ie/v18n78/1665-2673-ie-18-78-193.pdf

Cedeño, E., \& Moya, E. (2019). La retroalimentación como estrategia de mejoramiento del proceso $\begin{array}{lllll}\text { formativo de } & \text { los } & \text { Recuperado }\end{array}$ https://www.eumed.net/rev/atlante/2019/08/retroalimentacioneducandos.html

Contreras, G., \& Zuñiga, C. (2017). Concepciones de profesores sobre retroalimentación: una revisión de la literatura. Recuperado de http://repositorio.unab.cl/xmlui/bitstream/handle/ria/4277/ContrerasPerez_G_Concepciones_ de_profesores.pdf?sequence=1\&isAllowed=y

Domínguez, S. (2010). La Educación, cosa de dos: La escuela y la familia. Recuperado de: https://www.feandalucia.ccoo.es/docu/p5sd7214.pdf

Flórez, G. (2017). El acompañamiento familiar en el proceso de formación escolar para la realidad colombiana: de la responsabilidad a la necesidad. Recuperado de: file://C:/Users/ROBERTO/Downloads/DialnetEIAcompanamientoFamiliarEnEIProcesoDeFormaci onEsco5893097\%20(2).pdf

Frontela, B. (2013). El docente en Educación Infantil. Recuperado de https://uvadoc.uva.es/bitstream/handle/10324/4801/TFGL280.pdf;jsessionid=DF9EA2D04E87D 23C4DBB8990B7AE41EC?sequence=1

Gallego, A., Ospina, S., Quintero, S., \& Jaramillo, B. (2015). Una mirada hacia la consolidación de la educación preescolar y el saber pedagógico de los agentes educativos. Recuperado de file://C:/Users/Usuario/Downloads/629-2991-2-PB(1).pdf

Gil, M., \& Sánchez, O. (2004). Educación Inicial o Preescolar: El niño y la niña menores de tres años. Algunas orientaciones a los docentes. Recuperado de https://www.redalyc.org/pdf/356/35602713.pdf 
Gómez, E., Navas, D., Aponte, G., \& Betancourt, L. (2014). Metodología para la revisión bibliográfica y la gestión de información de temas científicos, a través de su estructuración y sistematización. Recuperado de https://www.redalyc.org/pdf/496/49630405022.pdf

Gómez, H. (2014). El aprendizaje activo como mejora de las actitudes de los estudiantes hacia el aprendizaje.

Recuperado

de https://academicae.unavarra.es/bitstream/handle/2454/9834/TFM\%20HELENA\%2OSIERRA.pdf

León, L. (2013). Rol del docente inicial en el desarrollo físico y social del niño de primera infancia. Recuperado de http://biblio3.url.edu.gt/Tesario/2013/05/09/De\%20Leon-Leslie.pdf

Lima, G. (2017). Enriquecer la realimentación para consolidar aprendizajes. Recuperado de: file://C:/Users/ROBERTO/Downloads/17328-Texto\%20del\%20art\%C3\%ADculo-48104-1-1020170626.pdf

Llerena, M., \& Sánchez, I. (2020). La retroalimentación y la consolidación de aprendizajes en procesos en niños y niñas de Maternal 1 y 2 del Centro de Desarrollo Infantil Universitario UTA. https://repositorio.uta.edu.ec/jspui/handle/123456789/30902

Martínez, G., Guevara, A., \& Valles , M. (2016). El desempeño docente y la calidad educativa. Recuperado de https://www.redalyc.org/pdf/461/46148194007.pdf

Osorio, K., \& Alexis, L. (2014). La Retroalimentación Formativa en el Proceso de EnseñanzaAprendizaje de Estudiantes en Edad Preescolar. Recuperado de file://C:/Users/Usuario/Downloads/DialnetLaRetroalimentacionFormativaEnEIProcesoDeEnsen anza-4704214(1).pdf

Ospina, M. (2015). El juego como estrategia para fortalecer los procesos básicos de aprendizaje en el nivel preescolar. Recuperado de: http://repository.ut.edu.co/bitstream/001/1576/1/Trabajo\%20de\%20Grado\%20\%20Maria\%200spina\%20version\%20aprobada.pdf

Polanco Hernández, Ana (2004). La pregunta pedagógica en el nivel inicial. Revista Electrónica "Actualidades Investigativas en Educación" Recuperado de: https://www.redalyc.org/articulo.oa?id=447/44740213

Razeto, A. (2016). El involucramiento de las familias en la educación de los niños. Cuatro reflexiones para fortalecer la relación entre familias y escuelas. Recuperado de http://www.scielo.edu.uy/pdf/pe/v9n2/v9n2a07.pdf 
Rodríguez, E. (2006). Los padres de familia y su participación en el aprendizaje de sus hijos. Recuperado de: http://200.23.113.51/pdf/23757.pdf

Silva, P. (2014). Aprendizaje significativo, conocimientos previos y categorías didácticas para la enseñanza de la historia: consideraciones y presencias en dispositivos didácticos y trabajo docente. Recuperado de: http://bibliotecadigital.academia.cl/bitstream/handle/123456789/3226/TPHIS\%20135.pdf?sequ ence $=1 \&$ isAllowed $=\mathbf{y}$.

Trujillo, Z., Henriquez, D., \& Labrador, M. (2013). La Consulta Docente en la consolidación del aprendizaje. Una propuesta de estrategia didáctica. Recuperado de http://scielo.sld.cu/scielo.php?script=sci_arttext\&pid=S1561-31942013000500012

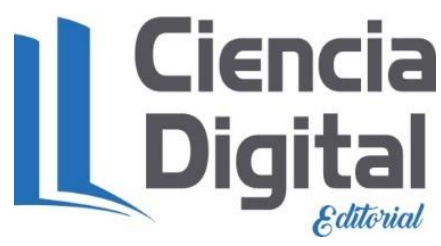


PARA CITAR EL ARTÍCULO INDEXADO.

Llerena Espín, A. M., Sánchez Fernández, I., \& Hernández Navarro, Y. (2020). La retroalimentación y su influencia en la consolidación de aprendizajes en proceso, de niños y niñas $\begin{array}{llll}\text { de } & \text { Educación } & \text { Inicial. } & \text { ConcienciaDigital, }\end{array}$ https://doi.org/10.33262/concienciadigital.v3i3.2.1408

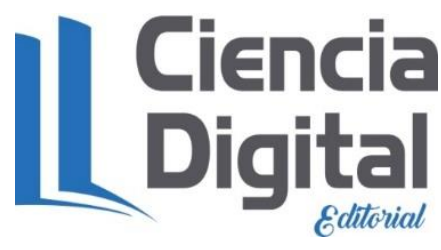

El artículo que se publica es de exclusiva responsabilidad de los autores y no necesariamente reflejan el pensamiento de la Revista Conciencia Digital.

El artículo queda en propiedad de la revista y, por tanto, su publicación parcial y/o total en otro medio tiene que ser autorizado por el director de la Revista Conciencia Digital.
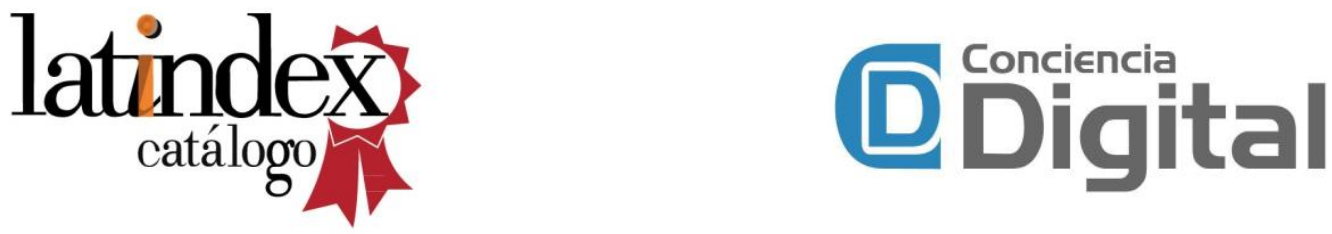